\title{
Commentary: This is your brain, and this is your brain on deep hypothermic cardiac arrest
}

James Jaggers, MD

\author{
From the Department of Surgery, University of Colorado, Aurora, Colo; and Department of Congenital Cardiac \\ Surgery, Childrens Hospital Colorado, Aurora, Colo. \\ Disclosures: Author has nothing to disclose with regard to commercial support. \\ Received for publication March 17, 2019; accepted for publication March 18, 2019. \\ Address for reprints: James Jaggers MD, Childrens Hospital Colorado, 13123 E 16th Ave, Aurora CO 80045 \\ (E-mail: James.Jaggers@Childrens.Colorado.org). \\ J Thorac Cardiovasc Surg 2019;158:893-4 \\ $0022-5223 / \$ 36.00$ \\ Copyright (C) 2019 Published by Elsevier Inc. on behalf of The American Association for Thoracic Surgery \\ https://doi.org/10.1016/j.jtcvs.2019.03.080
}

Since the inception of cardiac surgery, we have been debating the relative benefit and risk incurred with the use of deep hypothermic cardiac arrest (DHCA) in the surgery for complex congenital cardiac defects. The study in this issue of the Journal by $\mathrm{Tu}$ and colleagues ${ }^{1}$ brings us to the current era of complex and sophisticated genetic analysis in analysis of the cellular response. This elegant large animal experimental model characterizes the genetic response of the striatum of the neonatal piglet brain after exposure to cardiopulmonary bypass (CPB) and DHCA. The animals were humanely killed, and samples of the piglet striatum were harvested. Whole transcriptome shotgun sequencing was used to obtain a global genomic fingerprint of the striatum in response to CPB and DHCA.

For those of you who either cannot remember neuroanatomy or, like me, never learned, the striatum is composed of 3 nuclei: the caudate nucleus, the putamen, and the ventral striatum. This area of the subcortical brain serves as the primary input to the rest of the basal ganglia system and coordinates multiple aspects of cognition, including both motor and action planning, decision making, motivation, reinforcement and reward, and perception. It is also the part of the brain to which damage may result in the long-term neurodevelopmental abnormalities seen in children with complex congenital heart defects.

This study uses a powerful tool called PANTHER, (Protein Analysis Through Evolutionary Relationship). This powerful technique can classify differentially expressed genes according to ontology, thus inferring their function, pathways, and biologic processes on the basis of their evolutionary relationships to genes with known functions. $\mathrm{Tu}$ and colleagues ${ }^{1}$ found significant upregulation of genes involved in inflammatory and apoptotic pathways, with several genes overlapping. This is consistent with many other experimental studies, which have demonstrated profound inflammatory changes in the brain after DHCA. Another finding in this study was that several genes that are involved in cell growth and proliferation and cell

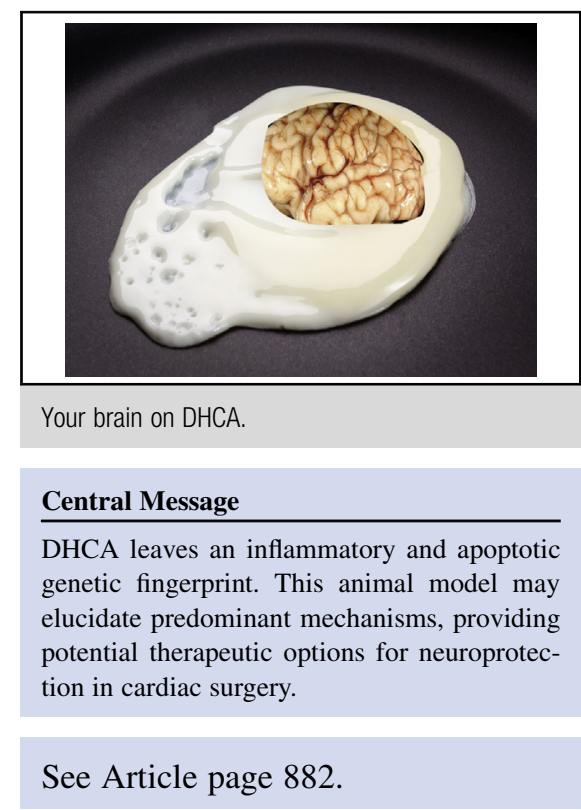

adhesion were downregulated, indicating apoptotic injury. Not surprisingly, nuclear factor $\kappa \mathrm{B}$ target genes accounted for $63 \%$ of the upregulated genes. Nuclear factor $\kappa \mathrm{B}$ is a ubiquitous transcription factor associated with chemokine, cytokines, and other inflammatory pathways, and it has been implicated in ischemia-reperfusion injury as well.

Although this is an elegant experimental study that uses the latest technology for gene analysis, we must keep in mind that much of the risk for neurologic injury and neurodevelopmental deficits seems to be more the result of impaired fetal brain development and circulation, coexisting genetic syndromes, and presurgical and postsurgical hemodynamic instability, rather than the insult of CPB with or without DHCA. So, although DHCA and CPB are "modifiable" risk factors, much work remains to investigate the other important factors associated with neurodevelopmental abnormalities.

An important limitation of this study, which Tu and colleagues ${ }^{1}$ acknowledge in the final article, is the lack of an appropriate control group. It is difficult to know the relative contributions of DHCA, hypothermia, and CPB to the potential injury. It may also be that the sensitivity of this technique is picking up gene regulation changes that may not have substantial clinical implications. It might also be fascinating to compare mature pigs with neonatal piglets, because there may be substantial difference in the gene transciptome. I look forward to future investigations by this group, which I hope not only will characterize the 
contribution of CPB alone but also will evaluate the potential neuroprotective strategies, such as intermittent cerebral perfusion and selective antegrade cerebral perfusion, as well as the potential benefit of ischemic preconditioning.

\section{Reference}

1. Tu LN, Timms AE, Kibiryeva N, Bittel D, Pastuszko A, Nigam V, et al. Transcriptome profiling reveals activation of inflammation and apoptosis in the neonatal striatum after deep hypothermic circulatory arrest. J Thorac Cardiovasc Surg. 2019;158:882-90.e4. 